\title{
Loss of 908 Second Molars in Chinese adults: Consequences of the Presence of Their Neighboring Third Molars?
}

\section{Li-Juan Sun}

Fourth Military Medical University School of Stomatology

\section{Yi Tian}

Fourth Military Medical University School of Stomatology

Hong-Lei Qu

Fourth Military Medical University School of Stomatology

\section{Yang Yang}

Fourth Military Medical University School of Stomatology

Fa-Ming Chen ( $\square$ cfmsunhh@fmmu.edu.cn )

Fourth Military Medical University School of Stomatology https://orcid.org/0000-0002-8398-2104

\section{Research article}

Keywords: Second molar, Tooth loss, Tooth extraction, Third molar

Posted Date: May 6th, 2020

DOI: https://doi.org/10.21203/rs.3.rs-26351/v1

License: () (i) This work is licensed under a Creative Commons Attribution 4.0 International License. Read Full License 


\section{Abstract}

Background Many studies have reported that the presence of third molars (M3s) can increase the risk of pathology in neighboring second molars (M2s). However, whether the presence of M3s can effect the mortality of M2s remains unknown. The main aim of this study was to reveal the reasons for M2 loss and how the M2 loss were related to their neighboring M3s. Methods This was a retrospective cross-sectional study. A review of the medical records and radiographic imagines of patients who had their M2(s) removed was undertaken to analyze the reasons for tooth extraction and how those reasons were related to the presence of M3s situated adjacent to the removed M2s.

Results Clinical material (from January to March 2019) of 800 patients met the inclusion criteria were involved into this study. M2s in 908 quadrants (i.e. 908 M2s) were removed from these enrolled patients, wherein 382 quadrants were with the absence of M3s and the other 526 quadrants were with the presence of M3s. The average age of patients whose missing M2s had or had no neighboring M3s was $52.4 \pm 14.8$ years and $56.7 \pm 14.9$ years respectively (Mann-Whitney $U$ test, $p<0.001$ ). When the reasons for the 908 extracted M2s were traced, it was found that 433 teeth $(47.7 \%)$ were removed due to caries and sequelae, while 300 teeth (33.0\%) were lost for periodontal diseases. In addition, substantial evidence confirmed that $14.4 \%$ of the M2s with adjacent M3s were removed due to diseases mainly in the distal surface which were closely related to their neighboring M3s, and it was much higher than that was identified from removed M2s without adjacent M3s (1.8\%). Except for residual roots, 42.2\% M3s were removed along with neighboring M2s simultaneously.

Conclusions The presence of M3s, no matter impacted or erupted M3s, was associated with an earlier loss of their neighboring M2s.

\section{Background}

Tooth loss is a global problem which not only impacts patients' daily life, but also be associated with various systemic diseases [1, 2, 3]. Clinical evidence suggests that, except for third molar (M3), second molar (M2) is one of the most frequently removed permanent teeth in adults [4,5]. Similar to other teeth, many factors may affect a M2's life span, such as oral hygiene, occlusal condition, trauma, smoking, etc. [6]. Among the various risk factors to M2 pathology, the presence of its neighboring M3s has attracted increasing attention $[7,8,9]$. In this context, studies have confirmed that the presence of impacted M3s (I-M3s) increases the risk of M2 pathology by $1.45-4.88$ folds [8, 9]. In recent years, investigations have found that erupted M3s (E-M3s) also negatively impact oral health and scientists have reported that the presence of E-M3s increases the risk of M2 pathology by $1.44-1.77$ folds $[8,9,10]$. When M2s are too heavily damaged to be treated or controlled, they can only be removed.

To protect M2s from loss, on one hand we should try to identify early pathology (i.e. caries and periodontitis) before irreversible damage occurs in M2s, and on the other hand, minimizing the risk factors that cause M2 caries and periodontitis is of equal importance. With regard to the well-known risk factor - the presence of M3s, preventive extraction of M3s may be the most thoroughly solution. There is substantial evidence that the removal of M3s leads to a lower incidence rate of distal surface caries on M2s and improves the 
periodontal health of distal M2s $[11,12,13,14]$. However, the decision on preventive extraction of asymptomatic M3s is challenging for dentists as well as patients. In many clinical situations, asymptomatic M3s are indeed more likely retained until irreversible damages to their neighbors occur $[15,16,17]$.

Unfortunately, researchers have found that, even the health status of M2s is actively monitored, there is still a significant rate of M2 mortality [18]. Till now, we still don't know whether there is a relationship between M2 loss and their adjacent M3s. If the M2s are removed due to the presence of M3s, maybe it is time to justify whether medical decision based on the high confidence to our patients' compliance is wise.

Tracing the reasons of M2 loss in clinics may help dentists better understand the relationship between M2 loss and their neighboring M3s and hence to do timely interventions to actively protect M2s from M3-related damage. In this study, 800 patients with their $908 \mathrm{M} 2 \mathrm{~s}$ being removed were analyzed to identify (1) who's M2s were removed; (2) the reasons why M2s were extracted and to what degree those reasons were closely related to the presence of their neighboring M3s; (3) how these M3s were handled in clinic.

\section{Methods}

\section{Ethics}

This was a retrospective cross-sectional study based on the medical records and radiographic imagines of patients who had their M2(s) removed in the Dental Hospital of Fourth Military Medical University (FMMU), Xi'an, China. The research process was strictly followed the requirements of the Helsinki declaration and was approved by the ethics committee of this hospital.

\section{Inclusion and exclusion criteria}

Clinical material of patients who had visited the Department of Oral and Maxillofacial Surgery from January 2019 to March 2019 were continuously screened. The inclusion criteria were: (i) patients with age $\geq 18$ years old; (ii) patients who have at least one M2 extracted; (iii) patients with complete and explicit medical records and radiographic imagines of their extracted M2s (prior to extraction) and their neighboring M3s (if any). Patients with incomplete medical records or inadequate quality of radiographic imagines on either the extracted M2 or their neighboring M3 had incomplete medical records or inadequate quality of radiographic imagines were excluded from this study. Besides, patients with discrepant medical records and radiographic imagines also excluded from analysis.

\section{Reasons for M2 extraction}

The reasons for M2 extraction were mainly classified into the following three categories: (i) Severe caries and sequelae - whenever the primary indication for extraction was caries or its sequelae, e.g., untreatable caries, residual roots, diseases of pulp and periapical tissue; (ii) Irreversible periodontitis - when M2 was extracted for periodontal breakdown; (iii) Others - Orthodontic needs, cysts or tumors, non-carious defect (e.g., tooth fracture or root resorption), and other ambiguous reasons for $\mathrm{M} 2$ extraction. When periodontal disease and caries (or its sequelae) presented in a M2 at the same time, only the predominant reason for M2 extraction was recorded. In this case, if it is not possible to identify which disease was more likely the main reason for M2 loss, it was recorded as comorbidities and assigned to the category of "others". 


\section{Data collection}

Demographic information of patients (age, gender, systematic diseases, smoking status, etc.) and details about the extracted M2s (prior to extraction) and their neighboring M3s (disease and clinical situation of the M2s before extraction, the status of M3s, etc.) were continuously extracted from the electronic medical system (doctors) of the Hospital. To minimize errors, the radiographic images were observed in a digital viewer (Hi Net, Hwatech, Xi'an, China) to judge whether findings from the radiographic images were consistent with the data in medical records (especially the reasons of M2 extraction).

\section{Groups}

Based on the absence/presence and status of M3s, quadrants with M2s being extracted were divided into M3 $(-)$ group (quadrants with M2s being extracted with the absence of M3s) and M3 (+) (quadrants with M2s being extracted with the presence of M3s). In this context, quadrants in M3 (+) group were further divided into E-M3 group (quadrants with M2s being extracted with erupted M3s) and I-M3 group (quadrants with M2s being extracted with impacted M3s), wherein quadrants with M2s being extracted with neighboring M3s that were residual roots or microdontia were excluded for analysis.

\section{Statistical methods}

EpiData (version 3.0, the EpiData Association, Denmark) was used to collect data, and SPSS (version 20, IBM, Chicago, USA) was used to analysis data. Missing or abnormal data were replenished according to patients' medical records and radiographic images. The normality of qualitative data (age) was tested by Shapiro-Wilk test. Results showed that the age of all groups was non-normally distributed. Therefore, the differences of age between two groups were tested by Mann-Whitney $U$ tests. The differences of quantitative data (gender, jaw, right/left and indications) between two groups were tested by Chi-Square tests. The two-sided significant level was set at $p<0.05$.

\section{Results}

\section{Clinical material for analysis}

According to the electronic medical records system, approximately twenty thousand person-time visited the Department of Oral and Maxillofacial Surgery from January to March in 2019. Among them, there were 854 patients who had at least one M2 removed. Clinical material of 54 patients was excluded to this study due to either incomplete medical records or poor quality of radiographic images or both reasons. Finally, Clinical material of 800 patients was applied for analysis in this study.

\section{Characteristics of the patients}

Characteristics of the patients whose clinical material was applied for investigation were shown in Table 1. Most of the subjects (87.6\%) had only one M2 removed. The age of these enrolled patients ranged from 19 years to 91 years and the mean age was 54.1 (SD: 15.0) years. Of all the subjects, $95.3 \%$ were nonsmokers. The proportion of males (54.6\%) was higher than that of females (45.4\%). In addition, the 
prevalence of systematic disease(s) in these patients was $36.0 \%$, and among them, $36.1 \%$ suffered a combination of several systematic diseases.

Table 1

Characteristics of the patients whose clinical material was applied for analysis $(N=800)$

\begin{tabular}{|c|c|c|c|}
\hline \multicolumn{2}{|l|}{ Characteristics } & \multirow{2}{*}{$\begin{array}{l}\boldsymbol{N} \\
54.1 \pm\end{array}$} & \multirow{2}{*}{$\begin{array}{c}\% \\
5.0\end{array}$} \\
\hline \multirow[t]{2}{*}{ Age (years) } & Mean \pm SD & & \\
\hline & Median (Q25, Q75) & \multicolumn{2}{|c|}{$55.0(44.0,64.8)$} \\
\hline \multirow[t]{2}{*}{ Gender } & Male & 437 & 54.6 \\
\hline & Female & 363 & 45.4 \\
\hline \multirow[t]{2}{*}{ Systematic disease(s) } & No & 512 & 64.0 \\
\hline & Yes & 288 & 36.0 \\
\hline \multirow[t]{2}{*}{ Smoking } & No & 762 & 95.3 \\
\hline & Yes & 38 & 4.8 \\
\hline \multirow[t]{4}{*}{ Distribution of extracted M2s in patients } & 1 & 701 & 87.6 \\
\hline & 2 & 91 & 11.4 \\
\hline & 3 & 7 & 0.9 \\
\hline & 4 & 1 & 0.1 \\
\hline
\end{tabular}

Clinical material of the 800 patients (908 quadrants with 908 M2s being extracted) were applied for analysis in a quadrant (tooth)-level. In general, M2 extraction was more likely occurred between 50 and 59 years old and two-thirds of M2s were removed between the age of 40 and 69 years. Compared to females $(44.6 \%)$, more male patients (55.4\%) had their M2s extracted. Slightly over half of extractions were undertaken in the mandibular (51.7\%) region. There was no difference in M2 extraction between left side (49.8\%) and right side (50.2\%). According to patients' medical records and radiographic images, only 209 of the $908(23.0 \%)$ M2s had been treated for diseases (caries, periodontal disease, prosthesis, etc.) before M2 extraction.

According to the absence or presence of adjacent M3s, the 908 quadrants with M2s being removed were divided into the M3 (-) group and the M3 (+) group; 382 quadrants were within the M3 (-) group and 526 quadrants were within the M3 (+) group. Table 2 showed the age and distribution of extracted M2s adjacent to different status of M3s. The average age of patients in the M3 (-) group was 56.7 (SD: 14.9) years, while in the M3 (+) group it was 52.4 (SD: 14.8) years. When the normality of age was tested by Shapiro-Wilk test, it was found that the age of M3 (-) group and M3 (+) group was non-normally distributed. The presence of M3s was associated with an earlier loss of their neighboring M2s (Mann-Whitney $U$ test, $p<0.001$ ). The distributions of quadrants with M2s being extracted were also different according to absence/presence and 
different status of M3s neighboring the extracted M2s. In the M3 (+) group, the proportion of male patients was $59.5 \%$, which was higher than that of the M3 (-) group (49.7\%); the difference was statistically significant $(p=0.003)$. Besides, in the M3 (+) group, there were more quadrants with M2s being extracted in the mandibular (56.8\%) than that in the maxillary, while in the M3 (-) group, the maxillary predominated inversely $(p<0.001)$. In addition, as for the distribution of quadrants with M2s being extracted in the left/right side, no statistical difference was found between the two groups $(p=0.229)$.

Except for 36 quadrants with M3s being residual roots or microdontia, the other 490 quadrants in the M3 (+) group were further divided into E-M3 group (quadrants with M2s being extracted with erupted M3s) and I-M3 group (quadrants with M2s being extracted with impacted M3s). The number of the two groups were 145 and 345 , respectively. The mean age of patients in the E-M3 group was 54.2 (SD: 14.3) years and that of the I-M3 group was 46.2 (SD: 14.4) years (Table 2). Patients who had M2s neighbored with I-M3s extracted were younger than those who had M2s neighbored with E-M3s extracted $(p<0.001)$. In addition, the distribution of quadrants with M2s being extracted in maxillary/mandibular were different according to different status of M3s. 82.1\% of extracted M2s in the I-M3 group were undertaken in the mandibular, which was much higher than that in the E-M3 group $(p<0.001)$. There was no statistical difference between the I-M3 group and the EM3 group in gender and side (right/left) ( $p=0.312$ and $p=0.559$, respectively).

Table 2

Characteristics of the quadrants with M2s being extracted $(n=908)+$

\begin{tabular}{|c|c|c|c|c|c|c|c|c|c|}
\hline & \multirow{2}{*}{$\begin{array}{l}\text { M3 } \\
(-) \\
\text { group }\end{array}$} & \multicolumn{7}{|c|}{ M3 (+) group } \\
\hline \multicolumn{2}{|c|}{ Characteristics } & & Total & $p$ & $\begin{array}{l}\text { I-M3 } \\
\text { group }\end{array}$ & $p$ & $\begin{array}{l}\text { E-M3 } \\
\text { group }\end{array}$ & $p$ & $p^{*}$ \\
\hline \multicolumn{2}{|l|}{$n$} & 382 & $526^{\#}$ & & 145 & & 345 & & \\
\hline \multicolumn{2}{|c|}{ Age (mean $\pm S D)$ (years) } & $\begin{array}{l}56.7 \\
\pm \\
14.9\end{array}$ & $\begin{array}{l}52.4 \\
\pm \\
14.8\end{array}$ & $\stackrel{<}{0.001}$ & $\begin{array}{l}46.2 \\
\pm \\
14.4\end{array}$ & $\begin{array}{l}< \\
0.001\end{array}$ & $\begin{array}{l}54.2 \\
\pm \\
14.3\end{array}$ & 0.024 & $\begin{array}{l}< \\
0.001\end{array}$ \\
\hline \multirow{2}{*}{$\begin{array}{l}\text { Gender } \\
(\%)\end{array}$} & Male & 49.7 & 59.5 & 0.003 & 56.6 & 0.162 & 61.4 & 0.002 & 0.312 \\
\hline & Female & 50.3 & 40.5 & & 43.3 & & 38.6 & & \\
\hline \multirow[t]{2}{*}{ Jaw (\%) } & Maxillary & 55.5 & 43.2 & ${ }_{0.001}^{<}$ & 17.9 & $<_{0.001}$ & 51.6 & 0.292 & $\begin{array}{l}< \\
0.001\end{array}$ \\
\hline & Mandibular & 44.5 & 56.8 & & 82.1 & & 48.4 & & \\
\hline \multirow{2}{*}{$\begin{array}{l}\text { Right/Left } \\
(\%)\end{array}$} & Right & 48.4 & 52.5 & 0.229 & 54.5 & 0.215 & 51.6 & 0.394 & 0.559 \\
\hline & Left & 51.6 & 47.5 & & 45.5 & & 48.4 & & \\
\hline
\end{tabular}


When the reasons for M2 extraction was traced (Fig. 1), nearly half of M2s (47.7\%) were extracted due to caries and indeed the consequences of M2 caries; among them 61.7\% M2s were residual roots. Other 33.0\% M2s were removed due to local advanced periodontitis and the remaining 19.3\% extractions were for other reasons including tooth fracture, comorbidities, cysts, tumors and so on. It was interesting that in the category of "others", non-carious tooth fracture was the most common reason and about 1/10 of the $908 \mathrm{M} 2 \mathrm{~s}$ were extracted for this reason.

The main reasons for extraction of the $908 \mathrm{M} 2 \mathrm{~s}$ were caries and its sequelae (47.7\%) and periodontal diseases (33.0\%) (Fig. 1). But the distribution of them (caries and sequelae, periodontal diseases and others) were not the same between M2s with/without neighboring M3s. Table 3 showed the reasons for M2 removal in these groups. Similar to the entire samples, the most common reason of M2 extraction in the M3 (+) group and the M3 (-) group was still the caries and its sequelae. However, the distribution of indications for M2 removal between the two groups was different $(p=0.003)$. The percentage of M2 loss due to periodontal diseases was higher in the M3 (+) group than that of the M3 (-) group. Besides, the prevalence of extracted M2s ascribe to periodontal diseases was higher in the E-M3 group (42.3\%) than that of the I-M3 group (29.7\%, $p=0.009$ ).

In order to study whether the M2 loss was related to their adjacent M3s, the M2s were divided into three categories according to the location of diseases in M2s: mainly occurred in the distal surface of M2s, mainly occurred in other surfaces except for the distal surface, and the diseases which were unable to confirm whether they mainly occurred in the distal surface or not (Table 3). On the whole, in the M3 (-) group, only $1.8 \% \mathrm{M} 2 \mathrm{~s}$ were extracted for diseases mainly occurred in the distal surface, however, it was much higher in the M3 (+) group (14.4\%). In the I-M3 group, 64 M2s (44.1\%) were extracted due to distal diseases, including 29 extractions caused by periodontal diseases, 24 extracted M2s due to caries and its sequelae, and $11 \mathrm{M} 2 \mathrm{~s}$ loss of root resorption, cyst or other diseases. In the E-M3 group, although the rate of M2 extraction due to distal diseases (3.5\%) was lower than that of the I-M3 group, it was still higher than that of M2s without neighboring M3s. 
Table 3

Reasons for M2 extraction in quadrants with absence or presence (impacted/erupted) of neighboring M3s ( $n$ $=908$ )

\begin{tabular}{|c|c|c|c|c|c|c|c|c|c|}
\hline \multirow{2}{*}{\multicolumn{2}{|c|}{ Groups }} & \multirow[t]{2}{*}{$n$} & \multicolumn{4}{|c|}{ Indication (\%) } & \multicolumn{3}{|c|}{$\begin{array}{l}\text { Location of main disease in } \\
\text { M2s }(n)\end{array}$} \\
\hline & & & $\begin{array}{l}\text { Caries } \\
\text { and } \\
\text { sequelae }\end{array}$ & $\begin{array}{l}\text { Periodontal } \\
\text { diseases }\end{array}$ & Others & $p$ & $\begin{array}{l}\text { Distal } \\
(\%)\end{array}$ & $\begin{array}{l}\text { Non- } \\
\text { distal }\end{array}$ & Uncertain \\
\hline \multicolumn{2}{|c|}{ M3 (-) } & 382 & 49.0 & 27.7 & 23.3 & Reference & $\begin{array}{l}7 \\
(1.8)\end{array}$ & 5 & 370 \\
\hline \multirow[t]{3}{*}{$\begin{array}{l}\text { M3 } \\
(+)\end{array}$} & Total & $526^{\#}$ & 46.8 & 36.9 & 16.3 & 0.003 & $\begin{array}{l}76 \\
(14.4)\end{array}$ & 19 & 431 \\
\hline & I-M3 & 145 & 46.9 & 29.7 & 23.4 & 0.892 & $\begin{array}{l}64 \\
(44.1)\end{array}$ & 6 & 75 \\
\hline & E-M3 & 345 & 43.2 & 42.3 & 14.5 & $<0.001$ & $\begin{array}{l}12 \\
(3.5)\end{array}$ & 13 & 320 \\
\hline \multicolumn{2}{|c|}{ Total } & 908 & 47.7 & 33.0 & 19.3 & - & $\begin{array}{l}83 \\
(9.1)\end{array}$ & 24 & 801 \\
\hline
\end{tabular}

\section{The rate and reasons of M3s extracted with neighboring M2s}

Among the 908 quadrants, there were 526 with the presence of neighboring M3s to the M2s being removed, wherein 7 had been removed 1-4 years prior to extraction of the M2s. Among the 519 retained M3s (I-M3s: 143; E-M3s: 340; residual roots: 36), 237 were extracted along with the surgeries for M2 extraction; the other $282 \mathrm{M} 3 s$ were retained at least before the time of their clinical material was applied to this study (Table 4). Further analysis indicated that approximately $2 / 3$ (64.3\%) of the I-M3s (92/143) were removed along with M2 extraction, while only $1 / 3(32.9 \%)$ of the E-M3 (112/340) and their adjacent M2s were simultaneously extracted.

Table 4

The rate of M3 removal in the M2 extraction surgery $(n=519)$

\begin{tabular}{|llllll|}
\hline M3 (+) group & $\boldsymbol{n}$ & \multicolumn{4}{c|}{ Neighboring M3s removed along with M2s } \\
\cline { 3 - 6 } & & Yes $(\boldsymbol{n})$ & $\%$ & No $(\boldsymbol{n})$ & $\%$ \\
\hline Total & 519 & 237 & 45.7 & 282 & 54.3 \\
\hline I-M3 & 143 & 92 & 64.3 & 51 & 35.7 \\
\hline E-M3 & 340 & 112 & 32.9 & 228 & 67.1 \\
\hline Residual roots & 36 & 33 & 91.7 & 3 & 8.3 \\
\hline
\end{tabular}


The reasons of M3 removal along with adjacent M2 extraction were further analyzed in Fig. 2; indications for extraction were generally classified into four categories: prophylactic extraction, caries and sequelae, periodontal diseases and others. Except for the 33 residual roots, 92 I-M3s and 112 E-M3s (Table 4) were enrolled in this analysis. Results showed that the distribution of $M 3$ removal were greatly different between IM3s and E-M3s group $(p<0.001)$. Most I-M3s $(81.5 \%)$ were disease-free or in the early stages of diseases and they were proactively removed during the neighboring M2 extraction surgeries; only $7.6 \% 1-\mathrm{M} 3$ s were removed due to caries and sequelae and 3.3\% I-M3s were extracted because of periodontitis (Fig. 2a). However, across the extracted E-M3s, 4/5 were removed due to irreversible diseases (Fig. 2b), wherein 64.3\% (72/112) of EM3s were extracted for periodontal diseases and $15.2 \%$ of E-M3s (17/112) were removed due to caries and sequelae. Among them, $18.8 \%$ of the E-M3s (21) were extracted prophylactically, and evidence showed that at least 12 of the $112 \mathrm{E}-\mathrm{M} 3 \mathrm{~s}$ were non-functional teeth.

\section{Discussion}

It's unfortunately to find so many M2s were extracted in clinic. The authors analyzed $908 \mathrm{M} 2 \mathrm{~s}$ extracted from 800 patients to reveal who's M2s were removed, why were M2s removed, what degree were M2 loss related with their neighboring M3s and how were the M3s handled. The results showed that patients with the presence of M3s, no matter with I-M3s or E-M3s, may loss their neighboring M2s earlier than those with the absence of M3s; the prevalence of M2 loss due to caries and/or periodontal diseases in the distal surface (which were closely related to their neighboring M3s) was much higher in quadrants with M3s than that of those without M3s; nearly 2/3 I-M3s and 1/3 E-M3s were also removed during the neighboring M2 extraction surgeries.

Tooth loss can not only affects the daily quality of life of patients, but is also associated with a variety of systemic diseases, even with patients' longevity $[1,2]$. Therefore, clarifying the patterns of tooth loss may help dentists protect teeth more efficiently. Caries and periodontal diseases are the two major causes of tooth loss reported in previous studies [21,22], and they were consistent with the main reasons for M2 loss in this study (Fig. 1). Meanwhile, what makes the M2 different from other teeth is the presence of neighboring M3s, which is the most frequently impacted tooth [23]. M3 related diseases includes pericoronitis, caries, cysts, tumors, as well as the destruction of adjacent M2s, etc. $[24,25,26]$. The influence of M3s on adjacent M2s is widely concerned. Studies have confirmed that the presence of M3s is a risk factor for adjacent M2s $[8,9,10]$. Thus, it's not rigorous to separate M2s with neighboring M3s when tracing the pattern of M2 loss.

In this study, the main reason for M2 extraction was caries and its sequelae and the next was the periodontal diseases, however, the distribution of the indications were different in different groups. In the I-M3 group, approximately $1 / 2 \mathrm{M} 2 \mathrm{~s}$ were extracted for caries and its sequelae, and $1 / 3 \mathrm{M} 2 \mathrm{~s}$ were removed due to periodontal diseases (Table 3). However, when M3s were erupted, the percentage of periodontal diseases were much higher and it almost caught up with the percentage of caries and its sequelae. In recent years, several studies have explored the negative effect of erupted M3s and found that the risk of periodontal destruction of M2s may increase 1.44-6.79 folds when erupted M3 present [8, 9, 20,27]. In this study, the high percentage of periodontal diseases in the E-M3 group also remained clinicians to focus not only on the caries but also on the periodontal diseases. 
There are too many factors affect a M2's life span $[6,19]$, that's why to date there is no direct evidence revealing the relationship of $\mathrm{M} 2$ loss with their neighboring M3s. It's generally believed that the negative effect of M3s mainly occurred in the distal M2s $[9,13,20]$, thus the prevalence of M2 loss due to diseases in the distal surface may reflect the influence of M3s on the adjacent M2s. On the basis of this assumption, we compared the incidence of distal diseases in extracted M2s adjacent to different status of M3s. In this study, when M2s were divided into different groups according to the status of neighboring M3s, the findings were interesting. Results (Table 3) showed that the prevalence of M2 loss due to diseases in the distal surface was higher in M2s with presence of adjacent M3s (14.4\%) than those without M3s (1.8\%), especially when M3s were impacted (44.1\%). Although the rate of M2 extraction due to distal diseases (3.5\%) was lower than that of the I-M3 group, the passive effect of erupted M3s on their neighboring M2s still needs attention. In a 25year corhort study, scientists reported that during the follow-up, 14.6-39.1\% M2s adjacent to impacted M3s and 3.8\% M2s neighbored with erupted M3s were removed, while none of the M2s with the absence of M3s were extracted [18]. Combined the two studies, the authors concluded that the presence of M3s may lead to the loss of M2s. Besides, in this study, the diseases in 4/5 M2s were too severe to confirm where they mainly occurred (Table 3), which means diseases in some of these M2s may originated from the distal surface. What's more, most of caries or periodontal diseases that occurred only in distal M2s can be treated, thus our study may underestimated the negative effect of M3s.

Although the presence of M3s is generally believed to increase the residual periodontal pockets and caries risks of M2s [24, 26, 28], the decision on preventive extraction of asymptomatic M3s is hard for dentists and patients $[17,29]$. Even in most studies, the preventive extraction of M3s can be benefit for the health of neighboring M2s $[11,13,14]$, in many clinical situations, asymptomatic M3s are indeed more likely retained until irreversible damages happen $[15,16]$. Such evidence were also found in this study (Table 4). Nearly $2 / 3$ IM3s and 1/3 E-M3s were removed during the neighboring M2 extraction surgeries, and among them, 81.5\% IM3s and only $18.8 \%$ E-M3s were prophylactically extracted even when their M2s were heavily damaged (Fig. 2). For patients with low compliance and high risks, early intervention like prophylactical extraction of M3s may be more beneficial for the health of adjacent M2s than reserved until severe diseases occur. It's time for a change.

Preventive extraction of M3s may be the most thoroughly way to protect M2s. However, M3 extraction is neither risk-free nor cost-free, especially for some of E-M3s whom still have occlusal function in mouth. Therefore, the prophylactic removal of all asymptomatic M3s is unreasonable, and it is important to screen out high-risk patients and M2s. Studies have found that men, old age and other factors can increase the risk of $M 2$ destruction [10,27,30]. Although the characteristics of the population included in this study could not verify the risk factors of $\mathrm{M} 2 \mathrm{~s}$, the mean age of patients were old, the percentage of males were higher than that of females (Table 1).

Many studies have confirmed that age is significantly associated with tooth loss [3, 4]. In our study, patients' mean age was 54.1 years and it was similar to that of the studies of all types of permanent tooth loss $[5,31]$. Compared with M2s without adjacent M3s, the mean age of M2 extraction was younger when M3 present (Table 2). Especially in M2s with neighboring impacted M3s, the average age of M2 removal had been advanced for a whole decade. The authors speculate that the presence of M3s had accelerated the

Page $10 / 16$ 
progression of diseases in neighboring M2s and this hypothesis can be explained by other studies. Compared with M2s without neighboring M3s, the retained impacted M3s have been confirmed to increase the risk of M2 pathology by $2.16-4.88$ folds [8].

As far as the authors know, this was the first time to investigate the relationship of M2 extraction and status of neighboring M3s, and provided a specific view on the effect of M3s. Due to the large number of outpatient patients, it was difficult to examine every patient who met the criteria at the same time, meanwhile, the electronic record system is relatively complete and follows the unified standards, so we conducted the retrospective cross-sectional study. Besides, imaging data were collected to ensure the accuracy of data. It was concluded that the presence of M3s may advanced the age of M2 extraction. Long-term retention of impacted M3s and erupted M3s may cause great harm to adjacent teeth.

There were also some limitations. It was hard to judge whether the missing M3s were congenital absent ones or those M3s had been removed before this investigation. If so, the influence of M3s would be underestimated. Second, when an M2 suffers severe periodontal disease, it's easy to fall off itself, so the actual percentage of M2 loss due to periodontal diseases may be higher than that in this study.

Previous studies found that the presence of M3s can increase risk of pathology in adjacent M2s [32, 33]. In this study, the mean age and reasons for $\mathrm{M} 2$ extraction were different in the three groups, only indicating that M3s may be related with M2 loss, and we still don't know the scale and degree of that influence. The results reminds clinicians to pay more attention to the negative influence of M3s, no matter the impacted M3s or erupted M3s, and to conduct timely clinical interventions before irreversible damages emerge in neighboring M2s.

\section{Conclusion}

Based on clinical material of 800 patients with M2s in 908 quadrants being removed, this cross-sectional study analyzed the average age of patients without or with M3s neighboring their lost M2s and the reasons why the $908 \mathrm{M} 2 \mathrm{~s}$ were removed. It is found that M3-associated caries and periodontitis were two main reasons to the loss of their adjacent M2s and the presence of M3s led to an earlier loss of their neighboring M2s. We conclude that the presence of M3s, no matter I-M3s or E-M3s, may accelerate the loss of their adjacent M2s. However, further high-quality study based on a large number of population across various region is required to justify the accuracy of this finding.

\section{Abbreviations}

M2: Second molar

M3: Third molar

I-M3s: Impacted third molars

E-M3s: Erupted third molars 
SD: Standard deviation

Q25: The upper quartile

Q75: The lower quartile.

\section{Declarations}

\section{Ethics approval and consent to participate}

The research process was strictly followed the requirements of the Helsinki declaration and was approved by the ethics committee of the Dental Hospital of Fourth Military Medical University (IRB-REV-2017034).

\section{Consent for publication}

Not applicable.

\section{Availability of data and materials}

The datasets used and/or analysed during the current study are available from the corresponding author on reasonable request.

\section{Competing interests}

The authors declare that they have no competing interests.

\section{Funding}

This study was financially supported by the Major Research Program of the National Natural Science Foundation of China (Beijing, Subproject No. 81991503).

\section{Authors' contributions}

L.-J. Sun and Y. Tian collected data and drafted the manuscript, and they contributed equally to this work. H.L. Qu and Y. Yang participated in the statistical analyses. F.-M. Chen was the main supervisor and initiator of this study. All authors helped with the study design and manuscript drafting. All authors approved the final version of the manuscript.

\section{Acknowledgements}

Not applicable.

\section{References}

1. Christian C, Abnet Y-L, Qiao, Sanford M, Dawsey Z-W, Dong PR, Taylor SD, Mark. Tooth loss is associated with increased risk of total death and death from upper gastrointestinal cancer, heart disease, and stroke in a Chinese population-based cohort. Int J Epidemiol. 2005;34(2):467-74. doi:10.1093/ije/dyh375. 
2. Gerritsen AE, Allen PF, Witter DJ, Bronkhorst EM, Creugers NH. Tooth loss and oral health-related quality of life: a systematic review and meta-analysis. Health Quality of Life Outcomes. 2010;8(1):126. doi:10.1186/1477-7525-8-126.

3. Saito S, Ohi T, Murakami T, Komiyama T, Miyoshi Y, Endo K, Satoh M, Asayama K, Inoue R, Kikuya M, Metoki H, Imai Y, Ohkubo T, Hattori Y. Association between tooth loss and cognitive impairment in community-dwelling older Japanese adults: a 4-year prospective cohort study from the Ohasama study. BMC Oral Health. 2018;18(1):142. doi:10.1186/s12903-018-0602-7.

4. Marcus SE, Drury TF, Brown LJ, Zion GR. Tooth Retention and Tooth Loss in the Permanent Dentition of Adults: United States, 1988-1991. J Dent Res. 1996;75(2_suppl):684-95. doi:10.1177/002203459607502s08.

5. Olley RC, Renton T. \& P. M. Frost. Observational study investigating tooth extraction and the shortened dental arch approach. J Oral Rehabil. 2017;44:610-6. doi:10.1111/joor.12523.

6. Saito M, Shimazaki Y, Fukai K, Furuta M, Aida J, Ando Y, Miyazaki H, Kambara M. Risk factors for tooth loss in adult Japanese dental patients: 8020 Promotion Foundation Study. J Investig Clin Dent. 2019;10(2):e12392. doi:10.1111/jicd.12392.

7. Marciani RD. Is there pathology associated with asymptomatic third molars? J Oral Maxillofac Surg. 2012;70(9 Suppl 1):15-9. doi:10.1016/j.joms.2012.04.025.

8. Nunn ME, Fish MD, Garcia RI, Kaye EK, Figueroa R, Gohel A, ... Miyamoto T. Retained asymptomatic third molars and risk for second molar pathology. J Dent Res. 2013;92:1095-9. doi:10.1177/0022034513509281.

9. Kindler S, Holtfreter B, Koppe T, Mksoud M, Lucas C, Seebauer C, ... Ittermann T. Third molars and periodontal damage of second molars in the general population. J Clin Periodontol. 2018;45:1365-74. doi:10.1111/jcpe.13008.

10. Qu HL, Tian BM, Li K, Zhou LN, Li ZB, Chen FM. Effect of asymptomatic visible third molars on periodontal health of adjacent second molars: A cross-sectional study. J Oral Maxillofac Surg. 2017;75:2048-57. doi:10.1016/j.joms.2017.04.006.

11. Leung WK, Corbet EF, Kan KW, Lo EC, Liu JK. A regimen of systematic periodontal care after removal of impacted mandibular third molars manages periodontal pockets associated with the mandibular second molars. J Clin Periodontol. 2005;32(7):725-31.

12. Toedtling V, Devlin H, O'Malley L, Tickle M. A systematic review of second molar distal surface caries incidence in the context of third molar absence and emergence. Br Dent J. 2020;228(4):261-6.

13. Passarelli PC, Lajolo C, Pasquantonio G, D'Amato G, Docimo R, Verdugo F, D'Addona A. Influence of mandibular third molar surgical extraction on the periodontal status of adjacent second molars. J Periodontol. 2019;90(8):847-55.

14. Sun LJ, Qu HL, Tian Y, Bi CS, Zhang SY, Chen FM. Impacts of non-impacted third molar removal on the periodontal condition of adjacent second molars. Oral Dis. 2020. doi:10.1111/odi.13314.

15. Song F, Landes DP, Glenny AM, Sheldon TA. Prophylactic removal of impacted third molars: an assessment of published reviews. Br Dent J. 1997;10(9):339-46. 182(. 
16. Mettes TG, Nienhuijs ME, van der Sanden WJ, Verdonschot EH, Plasschaert AJ. Interventions for treating asymptomatic impacted wisdom teeth in adolescents and adults. Cochrane Database Syst Rev. 2005;18(2):CD003879.

17. Ghaeminia H, Perry J, Nienhuijs ME, Toedtling V, Tummers M, Hoppenreijs TJ, Van der Sanden WJ, Mettes TG. Surgical removal versus retention for the management of asymptomatic disease-free impacted wisdom teeth. Cochrane Database Syst Rev. 2016;(8):CD003879.

doi:10.1002/14651858.CD003879.pub4.

18. Nunn ME, Fish MD, Garcia RI, Kaye EK, Figueroa R, Gohel A, Ito M, Lee HJ, Williams DE, Miyamoto T. Response to letter to the editor, "Retained asymptomatic third molars and risk for second molar pathology". J Dent Res. 2014;93(3):320-1. doi:10.1177/0022034513520327.

19. Jiang Y, Okoro CA, Oh J, Fuller DL. Sociodemographic and health-related risk factors associated with tooth loss among adults in Rhode Island. Prev Chronic Dis. 2013;10:E45. doi:10.5888/pcd10.110285.

20. Li ZB, Qu HL, Zhou LN, Tian BM, Chen FM. Influence of non-impacted third molars on pathologies of adjacent second molars: A retrospective study. J Periodontol. 2017;88:450-6. doi:10.1902/jop.2016.160453.

21. Dye B, Thornton-Evans G, Li X, lafolla T. Dental caries and tooth loss in adults in the United States, 2011-2012. NCHS Data Brief. 2015;(197):197.

22. Müller A, Hussein K. Meta-analysis of teeth from European populations before and after the 18th century reveals a shift towards increased prevalence of caries and tooth loss. Arch Oral Biol. 2017;73:7-15.

23. Ioannis G, Gisakis FD, Palamidakis, Eleftherios-Terry R, Farmakis G, Kamberos S, Kamberos. Prevalence of impacted teeth in a Greek population. J Invest Clin Dent. 2011;2(2):102-9. https://doi.org/10.1111/j.2041-1626.2010.00041.x.

24. Campbell JH. Pathology associated with the third molar. Oral Maxillofac Surg Clin North Am. 2013;25:110.

25. Mello FW, Melo G, Kammer PV, Speight PM, Rivero ERC. Prevalence of odontogenic cysts and tumors associated with impacted third molars: a systematic review and meta-analysis. J Craniomaxillofac Surg. 2019;47(6):996-1002.

26. Venta I, Vehkalahti MM, Huumonen S, Suominen AL. Signs of disease occur in the majority of third molars in an adult population. Int J Oral Maxillofac Surg. 2017;46:1635-40.

27. Li ZB, Qu HL, Zhou LN, Tian BM, Gao LN, Chen FM. Nonimpacted third molars affect the periodontal status of adjacent teeth: A cross-sectional study. J Oral Maxillofac Surg. 2017;75:1344-50. doi:10.1016/j.joms.2017.02.005.

28. Ventä I, Vehkalahti MM, Suominen AL. What kind of third molars are disease-free in a population aged 30 to 93 years? Clin Oral Investig. 2019;23(3):1015-22.

29. Dodson TB, Susarla SM. Impacted wisdom teeth. BMJ Clin Evid. 2014;29:1302.

30. Soukaina Ryalat SA, AIRyalat Z, Kassob Y, Hassona, Mohammad H, Al-Shayyab, Sawair F. Impaction of lower third molars and their association with age: radiological perspectives. BMC Oral Health. $2018 \mathrm{Apr}$ 4;18(1):58. doi:10.1186/s12903-018-0519-1.

Page $14 / 16$ 
31. Jafarian M. \& A. Etebarian. Reasons for Extraction of Permanent Teeth in General Dental Practices in Tehran. IranMed Princ Pract. 2013;22:239-44. DOI:10.1159/000345979.

32. Venta I, Vehkalahti MM, Huumonen S, Suominen AL. Signs of disease occur in the majority of third molars in an adult population. Int J Oral Maxillofac Surg. 2017;46:1635-40.

doi:10.1016/j.ijom.2017.06.023.

33. Mello FW, Melo G, Kammer PV, Speight PM, Rivero ERC. Prevalence of odontogenic cysts and tumors associated with impacted third molars: A systematic review and meta-analysis. Journal of CranioMaxillofacial Surgery. 2019;47:996-1002. doi:10.1016/j.jcms.2019.03.026.

\section{Figures}

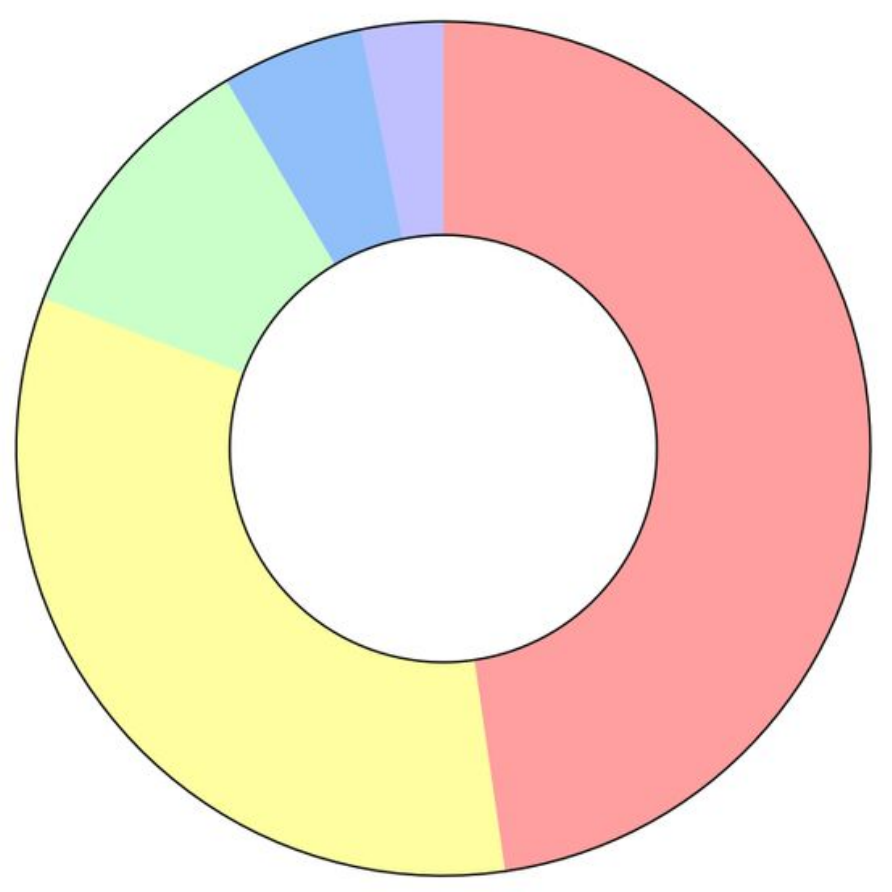

Caries and sequelae Periodontal diseases Tooth fracture Combined diseases Others

Figure 1

Reasons for M2 extraction in 908 quadrants 


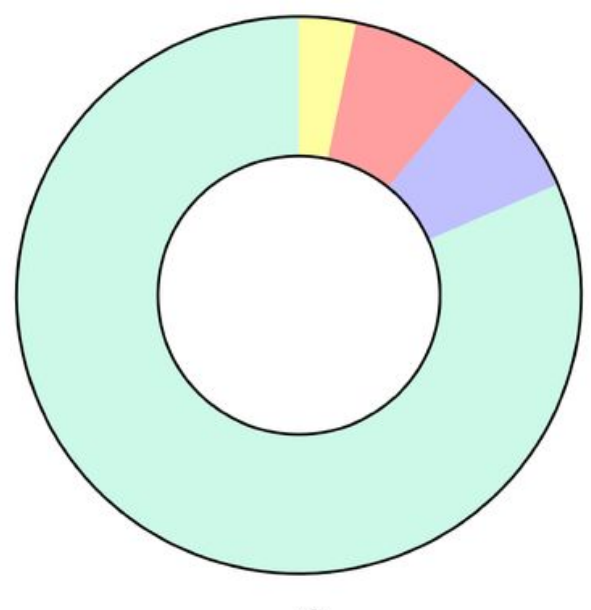

a

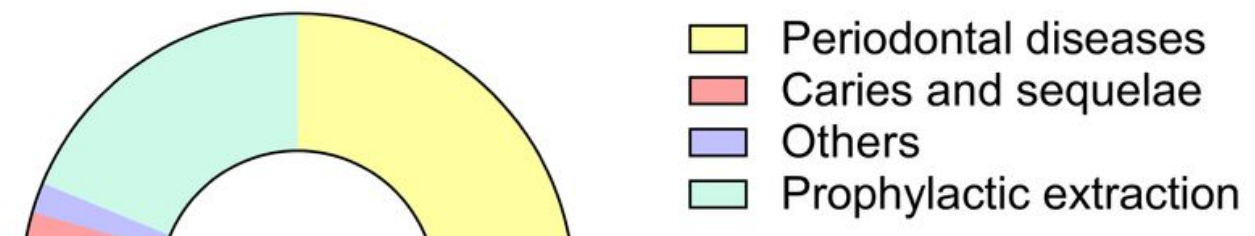

b

Figure 2

Reasons for extraction of I-M3 (a) and E-M3 (b) along with adjacent M2 extraction 